\title{
Réflexions sur quelques cas de charbon bactéridien "cryptique " chez des bovins
}

\author{
par A. PROVOST et M. TROUETTE
}

L'un de nous, inspecteur des abattoirs de FortLamy (Tchad) remarqua sur une carcasse de zébu Bororo, qui avait présenté une température de $40^{\circ}$ à l'examen sur pied, la présence de ganglions lomboaortiques de près de $20 \mathrm{~cm}$ de diamètre; il adressa le prélèvement au laboratoire pour recherche bactériologique.

Un simple frottis de la pulpe ganglionnaire, coloré au gram, montra un épais feutrage réalisé par l'enchevêtrement de longues chaînes de bacilles capsulés, gram-positifs, à extrémités typiques en « bambou» qui évoquaient d'emblée Bacillus anthracis.

L'ensemencement permit d'obtenir une culture pure de $B$. anthracis avec tous ses caracteres habituels. L'étude du pouvoir pathogène, aves une culture de 24 heures en bouillon, montra que les germes, à la dose de $1 \mathrm{~cm} 3$ de la dilution 10-6 par voie souscutanée, tuaient le cobaye en 24 heures et que les moutons inoculés succombaient en 36 heures après l'inoculation sous-cutanée de $1 \mathrm{~cm} 3$ de culture totale. Nous avions donc affaire à une souche très virulente.

Par contre, fait notable, la réaction de précipitation d'Ascoli, réalisée avec un exlrail de pulpe ganglionnaire était négative.

L'examen minutieux des autres carcasses du lot de bêtes abattues devait montrer de légères adénites pré-scapulaires, pré-crurales, ou mésentériques qui, dans ce pays profondément infesté de trypanosomes, n'auraient pas autrement semblé anormales, et à partir desquelles nous avons isolé le même germe Il convient d'insister sur le fait que ces zebus, de leur vivant, ne semblaient pas malades et ne présentaient même pas d'hyperthermie.

Des observations analogues devaient être faites à quelques mois de là et cette fois encore on pouvait isoler un $B$, anthracis virulent, mais toujours avec une réaction d'Ascoli négative.
Ces faits méritent quelques réflexions :

$1^{0}$ Au point de vue bactériologique : il est hors de doute que nous nous trouvons en présence d'une souche de $B$, anthracis, bien que la réaction d'Ascoli soit négative. La présence d'une capsule chez ce germe au stade parasitaire dans les humeurs de l'hôte, son immobilité, l'absence de fermentation de la salicine, permettent de le rattacher à la bactéridie charbonneuse, et de le séparer du groupe des anthracoides et des Bacillus. La virulence très grande du germe renforce encore cette opinion, quand on se rappelle que seul le bacille mésentérique s'est montré virulent, entre les mains de certains auteurs, par inoculation intra-péritonéale au cobaye d'une suspension dense.

Néanmoins la négativité de réaction d'Ascoli, réaction attribuée à la prćscnco du polyoside « $\mathrm{B}$ » d'Ivanovics précipitant avec un immunsérum, permet de penser que nous nous trouvons peut-être en présence d'une souche représentant une forme de transition entre les bacilles anthracoïdes (qui ne donnent pas de réaction) et $B$. anthracis. Quoiqu'il en soit, le pouvoir pathogène marqué du germe enlève tout intérêt pratique à une discussion dogmatique.

$2^{\circ}$ Au point de vue clinique et épidémiologique : Si ce n'est le premier cas signalé, les zébus ne semblaient pas souffrir de leur infection. Seul l'abatage devait la révéler. Rappelons que Nocard et Leclainche avaient déjà signalé que le charbon pouvait rester caché, cryptique, et la maladie ne se déclarer que lorsqu'une cause quelconque affaiblit l'animal. Besredka a fait des constatations semblables.

Les localisations mésentériques et lombo-aortiques d'une part, pré-scapulaires et pré-crurales d'autre part, permettent de supposer une contamination digestive ou cutanée sur quelque « champ-maudit». 
$3^{\circ}$ Au point de vue sanitaire : L'examen des animaux sur pied ne révélait rien de suspect. Toutes les carcasses avaient bien saigné; la rate était normale, le sang coagulait bien. Les seules lésions étaient ganglionnaires. La saisie totale fut la sanction de l'examen de laboratoire.

Mais le problème posé est grave en milieu tropical. Bien souvent, en effet, les animaux atteints de trypanosomoses ne presentent que de discrètes adénites, en tous points comparables à celles que nous avons évoquées. Il a fallu la découverte fortuite d'un germe charbonneux virulent pour éveiller l'attention. En de semblables circonstances, nombre d'inspecteurs n'auraient pas saisi les carcasses.
Qu'il nous soit donc permis d'insister, en conclusion, sur la gravité de ce problème en pathologie exotique : toute adénite suspecte doit immédiatement éveiller l'attention et provoquer le réflexe de consignation de la carcasse, en attendant le résultat de l'examen microscopique. Celui-ci n'offre aucune difficulté, même pour le vétérinaire broussard, toujours muni d'un microscope, car les bacilles sont faciles à voir sans coloration, dans un étalement de pulpe ganglionnaire. Ce simple geste peut éviter la maladie humaine, qui n'est pas rare dans nos régions.

\section{Laboratoire de l'Elevage, Fort-Lamy - Farcha, (Tchad).}

\section{SUMMARY}

Reflections on a case of "cryptic" anthrax in cattle.

The writers report the discovery of enlarged lomboaortic lymph nodes on some beef carcasses, at the Fort-Lamy slaughter-house. A microscopic examination showed long chains of capsulated bacilli very similar to B. anthracis. However, Ascoli's reaction was negative. Before slaughter, the animals did not appear to suffer from their infection. This discovery sets a serious problem in tropical quarters. Every doubtful adenitis should arouse attention and should provoke the consignation of the carcass, awaiting the result of the microscopic examination.

\section{RESUMEN}

Reflexiones sobre un caso de carbunco bacteridiano " criptico" en bovinos.

Los autores senalan el descubrimiento en el matadero, en esqueletos de bovinos, de ganglios lomboaórticos aumentados de volumen. El examen microscópico muestra largas cadenas de bacilos capsulados, muy parecidos al $B$. anthracis. Sin embargo, la reacción de Ascoli es negativa. Los aninales no parecen sufrir por la infección. Este descubrimiento plantea un grave problema en el medio tropical. Cualquier adenitis sospechosa debe Ilamar la atención y provocar la recogida del esqueleto hasta conocer el resultado del examen microscópico. 